\title{
Morbid Obesity with Achalasia: A Surgical Challenge
}

\author{
Monika E. Hagen • Micheal Sedrak • Oliver J. Wagner • \\ Garth Jacobsen • Mark Talamini • Santiago Horgan
}

Published online: 7 April 2010

(C) The Author(s) 2010. This article is published with open access at Springerlink.com

\begin{abstract}
Achalasia is a relatively rare medical condition that is classically not associated with obesity. The surgical treatment of a simultaneous occurrence of these two diseases requires careful consideration, and only a few reports can be found in the literature combining a Heller myotomy with gastric bypass, duodenal switch, or gastric banding. We report the case of a 69 -year-old female patient with early achalasia and obesity who underwent simultaneous laparoscopic gastric sleeve resection and robotic Heller myotomy. No intra- or postoperative complications occurred. A follow-up at 6 weeks showed a significant weight loss and resolved symptoms of achalasia. The case illustrates that a simultaneous gastric sleeve resection and robotic Heller myotomy might be an option for the treatment of concurrent obesity and achalasia.
\end{abstract}

Keywords Obesity · Achalasia · Myotomy · Gastric sleeve resection $\cdot$ Robotic surgery

\section{Introduction}

Achalasia is a relatively rare medical condition characterized by motor abnormalities of the esophagus resulting in stasis of ingested food [1]. Leading clinical symptoms include

M. E. Hagen $\cdot$ M. Sedrak $\cdot$ O. J. Wagner $\cdot$ G. Jacobsen $\cdot$

M. Talamini $\cdot$ S. Horgan

Center for the Future of Surgery, Division of Minimally

Invasive Surgery, University of California San Diego,

San Diego, CA, USA

M. E. Hagen $(\bowtie)$

Center for the Future of Surgery, Department of Surgery,

University of California San Diego,

200 West Arbor Drive,

San Diego, CA 92108, USA

e-mail: monikahagen@aol.com progressive dysphagia, regurgitation of food, retrosternal pain, and weight loss [2]. Therefore, achalasia is classically not associated with obesity. With obesity growing to epidemic proportions, the combination of these diseases may be seen in more frequency in the future. Generally, both achalasia and morbid obesity can be treated surgically in different approaches when occurring individually $[3,4]$, but concomitant treatment requires specific considerations: In that sense, surgical treatment of both problems in one patient will not to result in a good outcome unless they are addressed together. Successful therapy for achalasia usually leads to weight gain as the appetite of patients improve. Weight gain would be very detrimental in patients with morbid obesity and significant co-morbidities. On the other hand, a sole surgical approach addressing only the morbid obesity of this patient would not resolve the functional obstruction of the oesophagus. More frequent intake of highcalorie liquids would be the logical approach, but with counterproductive consequences and the success of weight loss surgery endangered. Therefore, surgical treatment should target to solve both problems at the same time and both surgical procedures should complement and not sabotage each other's effects. In addition to these functional considerations, the close proximity of a surgical approach to both achalasia and obesity complicate the approach. Only a few reports can be found on the concomitant performance of a Heller myotomy with gastric bypass, duodenal switch, or gastric banding [5-7].

\section{Case Report}

A 69-year-old female patient presented to the minimally invasive surgery clinic for evaluation of dysphagia to both liquids and solids. A manometry demonstrated a hypertensive lower oesophageal sphincter and esophageal dysmo- 


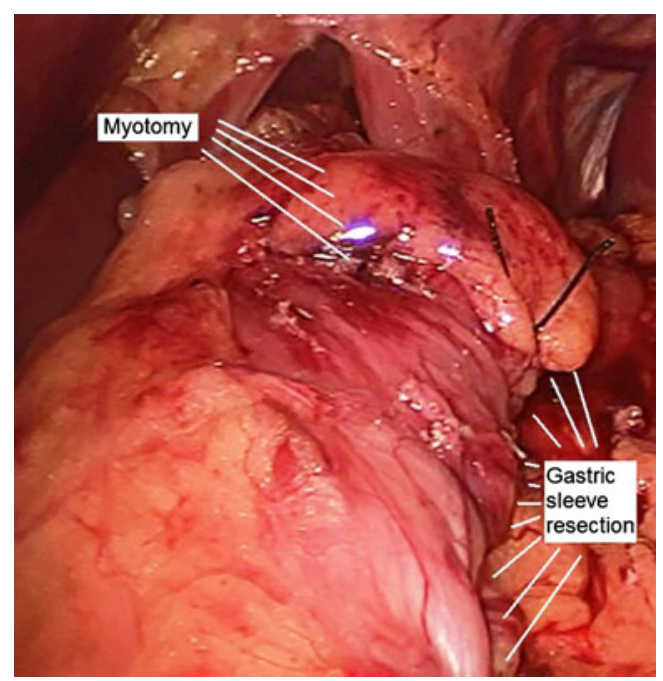

Fig. 1 Surgical field after robotic Heller myotomy and gastric sleeve resection

tility. Upper GI barium-enhanced radiographs revealed a very small mid-esophageal diverticulum with no evidence of a dilatated eosphagus. The patient's co-morbitities included obesity with a BMI of $40 \mathrm{~kg} / \mathrm{m}^{2}$, osteoarthritis, heart disease, hypertension, back pain, hypercholesterolemia, and depression. The patient had a previous knee replacement and back surgery. She used a cane or walker on wheels to walk and her overall health status made it hard for the patient to exercise. The patient reported moderate eating habits, but never felt full. She was previously successful at weight loss but never able to maintain a low weight.

With this clinical presentation, we decided to perform simultaneous surgeries for the patient's early achalasia and morbid obesity: The patient underwent robotic-assisted Heller myotomy and concomitant sleeve gastrectomy utilizing the same trocars for both procedures (Fig. 1; surgical field surgery). No intra- or postoperative complications occurred. The patient was discharged on postoperative day 4 on a liquid diet. Five weeks postoperatively, the patient stated that her symptoms of achalasia had resolved and that she had lost $11 \mathrm{lb}$.

\section{Discussion}

We decided to offer the patient with significant comorbidities one trip to the operating room, addressing both problems at the same time, in the most effective and least invasive way by utilizing the same trocar setup for both surgeries. Laparoscopic esophagogastric myotomy has been shown to be superior to other treatments for achalasia [8]. Robotic assistance for this procedure seems to result in safe outcomes with a reduced risk for perforation when compared to conventional laparoscopy [9]. Therefore, the decision to move forward with this treatment for our patient's early achalasia was clear. Finding the matching operation to address the patient's obesity was more difficult: Previous reports describe the successful combination of laparoscopic myotomy with gastric bypass, duodenal switch, and gastric banding [5-7]. However, we felt that gastric bypass and duodenal switch were not suitable due to their significant invasiveness and rate of complications for our patient with a great number of co-morbidities. Gastric banding is a less invasive method of weight loss surgery, but the circular restriction and the position in the upper part of the stomach could potentially interfere with the myotomy. Gastric sleeve resection has shown to be effective while less complicated when compared to gastric bypass and duodenal switch [10]. As laparoscopic gastric sleeve resection is becoming a standardized stand-alone bariatric procedure, it was our choice for this patient.

\section{Conclusion}

Finding the right treatment for concurrent obesity and achalasia appears to be a surgical challenge and only a few reports can be found in the literature. The positive short-term outcomes in our patient show that the simultaneous robotic Heller myotomy and laparoscopic gastric sleeve resection is a feasible option for the treatment of achalasia and morbid obesity. Weather this approach is superior to any other approach remains subject to further research.

Conflict Of Interest Disclosure M. Hagen, Micheal Sedrak, Oliver J. Wagner, Garth Jacobsen, Mark Talamini, S. Horgan: no conflict of interest

Open Access This article is distributed under the terms of the Creative Commons Attribution Noncommercial License which permits any noncommercial use, distribution, and reproduction in any medium, provided the original author(s) and source are credited.

\section{References}

1. Dughera L, Cassolino P, Cisarò F, et al. Achalasia. Minerva Gastroenterol Dietol. 2008;54(3):277-85.

2. Pohl D, Tutuian R. Achalasia: an overview of diagnosis and treatment. J Gastrointest Liver Dis. 2007;16(3):297-303.

3. Eckardt AJ, Eckardt VF. Current clinical approach to achalasia. World J Gastroenterol. 2009;15(32):3969-75.

4. Jaunoo SS, Southall PJ. Bariatric surgery. Int J Surg 2010;8(2): 86-9. Epub 2009 Dec 16. 
5. O'Rourke RW, Jobe BA, Spight DH, et al. Simultaneous surgical management of achalasia and morbid obesity. Obes Surg. 2007;17 (4):547-9.

6. Kaufman JA, Pellegrini CA, Oelschlager BK. Laparoscopic Heller myotomy and Roux-en-Y gastric bypass: a novel operation for the obese patient with achalasia. J Laparoendosc Adv Surg Tech A. 2005;15(4):391-5.

7. Jomni T, Dray X, Merrouche M, et al. Achalasia in an obese woman treated by laparoscopic gastric banding. Gastroenterol Clin Biol. 2008;32(11):973-5. Epub 2008 Oct 22.
8. Campos GM, Vittinghoff E, Rabl C, et al. Endoscopic and surgical treatments for achalasia: a systematic review and metaanalysis. Ann Surg. 2009;249(1):45-57.

9. Horgan S, Galvani C, Gorodner MV, et al. Robotic-assisted Heller myotomy versus laparoscopic Heller myotomy for the treatment of esophageal achalasia: multicenter study. J Gastrointest Surg. 2005;9(8):1020-9.

10. Arias E, Martinez PR, Ka ML, et al. Mid-term follow-up after sleeve gastrectomy as a final approach for morbid obesity. Obes Surg. 2009;19(5):544-8. 